\title{
Dynamic Aortic Aneurism Risk Factors
}

\author{
Oleg METSKER ${ }^{\mathrm{a}}$, Georgy KOPANITSA ${ }^{\mathrm{b}, 1}$, Olga IRTYUGA ${ }^{\mathrm{a}}$, Vladimir USPENSKIY ${ }^{\mathrm{a}}$ \\ a Almazov National Medical Research Centre, Saint-Petersburg, Russia \\ b ITMO University, Saint-Petersburg, Russia
}

\begin{abstract}
According to different systematic reviews incidence of thoracic aortic aneurysms (TAA) in the general population is increasing in frequency ranging from 5 to 10.4 per 100000 patients. However, only few studies have illustrated the role of different risk factors in the onset and progression of ascending aortic dilatation. Currently, noninvasive imaging techniques are used to assess the progression rate of aortic and aortic valve disease. Transthoracic (TT) Echocardiographic examination routinely includes evaluation of the aorta It is the most available screening method for diagnosis of proximal aortic dilatation. Since the predominant area of dilation is the proximal aorta, TT-echo is often sufficient for screening. We retrospectively analyzed the ECHO database with 78499 echocardiographic records in the Almazov National Medical Research Centre to identify patients with aneurysm. Detailed information including demographic characteristics, ECHO results and comorbidities were extracted from outpatient clinic and from hospital charts related to hospitalizations occurring within a year before index echocardiography was performed. Comorbid diseases were similarly extracted from outpatient clinic and/or hospital admissions. The classifier showed an AUC-ROC for predicting of aneurism detection after a repeated $\mathrm{ECHO}$ at $82 \%$.
\end{abstract}

Keywords. Aneurism, Machine learning, risk factor, prognosis

\section{Introduction}

Non-coronary heart diseases are a large heterogeneous group of diseases [1]. Given their prevalence and mortality in the general population, they have an important medical and social significance. We have chosen aortic valve and ascending aorta diseases as a model of non-coronary heart disease. These diseases are diagnosed most late due to the absence of clinical symptoms before the onset of complications up to lethal outcomes.

According to different systematic reviews incidence of thoracic aortic aneurysms (TAA) in the general population is increasing in frequency ranging from 5 to 10.4 per 100000 patients [2,3]. However, only few studies have illustrated the role of different risk factors in the onset and progression of ascending aortic dilatation [4,5]

Currently, noninvasive imaging techniques are used to assess the progression rate of aortic and aortic valve disease [6].

Transthoracic (TT) Echocardiographic examination routinely includes evaluation of the aorta. It is the most available screening method for diagnosis of proximal aortic dilatation. Since the predominant area of dilation is the proximal aorta, TT-echo is often sufficient for screening $[7,8]$.

${ }^{1}$ Corresponding Author, Georgy Kopanitsa, ITMO University, Saint-Petersburg, Russia, E-mail: georgy.kopanitsa@gmail.com 
Criteria for the progression of these diseases have not yet been developed. Both conservative and surgical therapies are not personalized. At the same time, the quality of life of patients can change rapidly depending on diagnosis, treatment and lifestyle.

The aim of this work is to identify anamnestic predictors of aortic aneurysm and to develop a model for identifying the need for re-diagnosis with ECHO.

\section{Methods}

We retrospectively analyzed the ECHO database with 78499 echocardiographic records in the Almazov National Medical Research Centre to identify patients with aneurysm. Detailed information including demographic characteristics, ECHO results and comorbidities were extracted from outpatient clinic and from hospital charts related to hospitalizations occurring within a year before index echocardiography was performed. Comorbid diseases were similarly extracted from outpatient clinic and/or hospital admissions.

\subsection{Inclusion criteria}

Age 18-75 years

Widening of the thoracic aorta $>40 \mathrm{~mm}$ and/ or hemodynamically significant impairment of aortic valve function (aortic valve velocity $>2.0$ and/ or degree 2 or greater aortic insufficiency)

Congenital aortic valve abnormality (bicuspid, monocuspid, quadricuspid aortic valve)

All patients underwent at least 2 comprehensive 2-dimensional and Doppler transthoracic echocardiography

\subsection{Data preprocessing}

We removed $1 \%$ of values having the highest $\mathrm{z}$-score to filter out some obvious outliers. Furthermore, we applied the min and max normalization to the remaining values.

\subsection{Classification model and feature importance}

To train a model for a prediction of the need of a second ECHO the experiment ran in the setting of stratified 5-fold cross-validation (i.e. random $80 \%$ of patients were used for training and $20 \%$ for testing target class ratios in the folds were preserved). A random forest algorithm was applied to calculate the feature importance. A correlation heatmap was used to analyze correlations between top predictors. The algorithm was implemented using Python 3.6.3 and the scikit-learn 0.19.12 library.

As an additional performance assessment score, we used AUC of ROC, which represents the trade-off between sensitivity and specificity of the model. The AUC was calculated based on an average of 5 curves (one curve per fold in the setting of 5 fold cross-validation).

\footnotetext{
${ }^{2}$ https://scikit-learn.org/stable/
} 
According to the results of the ECHO study, all patients can be divided into three groups:

Class 0. patients with normal aortic dimensions and low probability of aneurysm development, not requiring any intervention.

Class 1. patients with an existing or developing aortic aneurysm requiring treatment and dynamic monitoring according to current clinical guidelines.

Class 2. patients with normal-sized aorta, but at extremely high risk of aneurysm development in the coming years. In this group, strict control of cardiovascular risk factors and, possibly, certain drug interventions to prevent the disease seem to be rational;

\section{Results}

The classifier showed an AUC-ROC for predicting of aneurism detection after a repeated $\mathrm{ECHO}$ at $82 \%$. Features importance analysis is presented in the Figure 1.

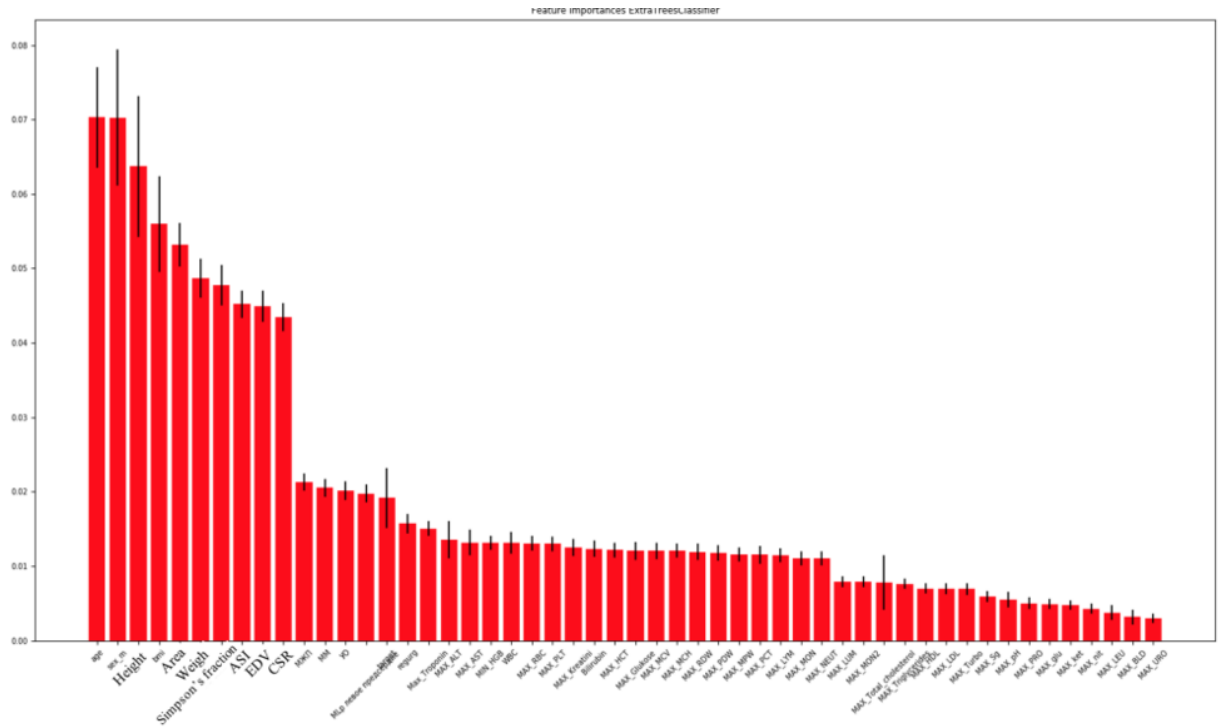

Figure 1. Feature's importance

The correlation matrix (Figure 2) demonstrates the fact that in the absence of structural changes in the ascending aorta, they are unlikely to develop in subsequent years in women, young adults, and low body weight. Interestingly, the likelihood of developing an aortic aneurysm is lower in patients with an existing aortic valve defect (stenosis or insufficiency). A Correlation matrix is presented in the Figure 2. 


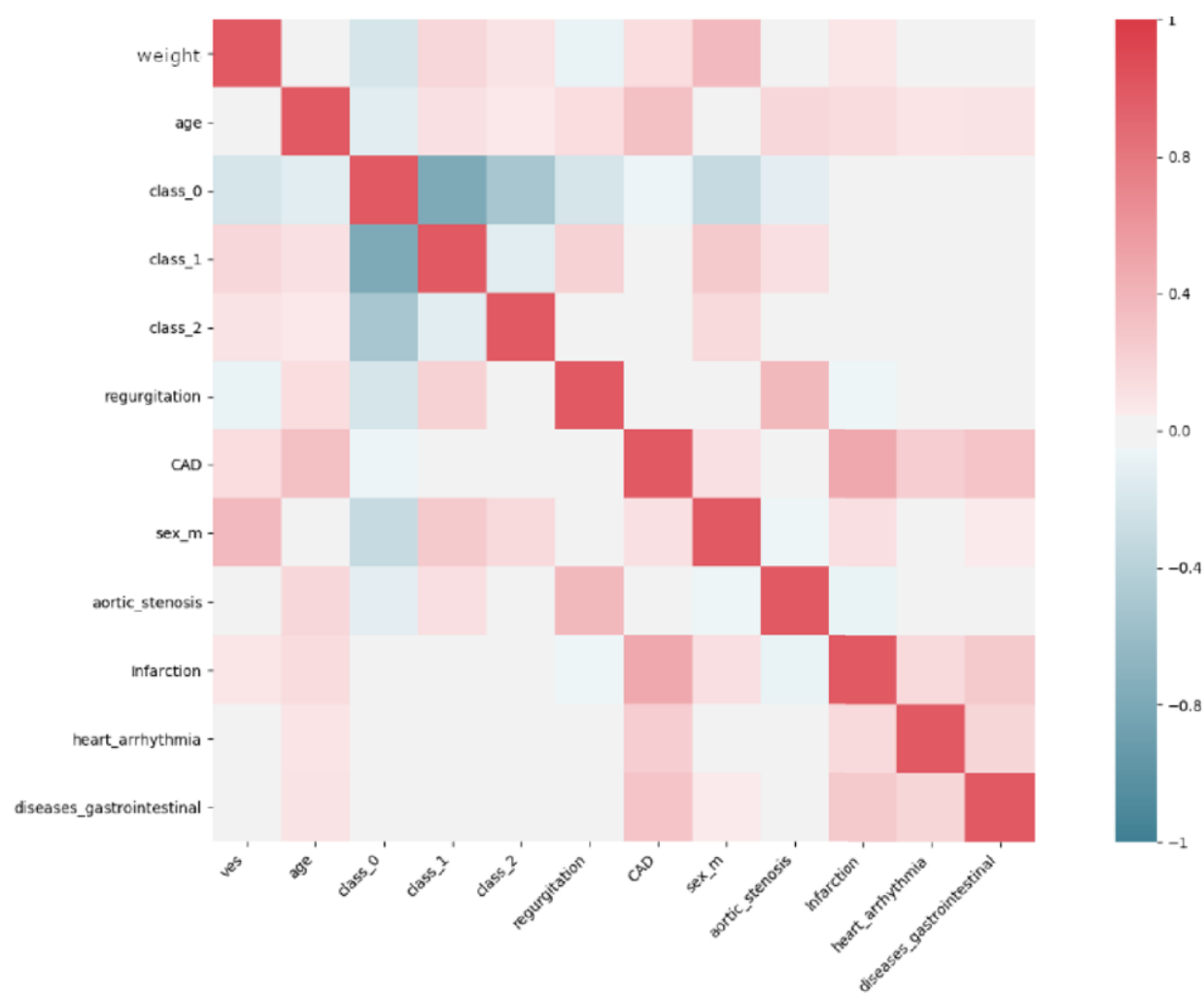

Figure 2. Heatmap

\section{Discussion}

Analyses of the findings confirm the significance of the known risk factors for aortic aneurysm development - age and male gender [3,5]. It should be noted that these risk factors are nonspecific, associated with the risk of a number of cardiovascular diseases, and, above all, atherosclerosis with lesions of various vascular basins and its acute complications, myocardial infarction and stroke [9]. Height is also a known risk factor for aortic aneurysm. In particular, it has been shown that height, rather than weight or body surface area, is most associated with the risk of aneurysm development [10]. It is possible that high height is more associated with connective tissue abnormalities associated with a high risk of aortic aneurysm formation, most pronounced in Marfan syndrome. The most significant appears to be the contribution of global left ventricular contractile function (Simpson ejection fraction). It has the leading position among all factors analyzed except for gender, age, height and body weight. Meanwhile, the risk of aneurysm development is associated with high contractile function of the left ventricle. It can be assumed that mechanical impact of blood flow on the aortic wall makes a significant contribution to the development of aortic aneurysm in predisposed patients with high cardiac contractile function. This is fundamentally different from the vast majority of patients with cardiovascular disease. For those an adverse prognosis is associated with decreased left ventricular contractile function, and low values of Simpson ejection fraction are considered as a universal marker of adverse prognosis [11]. 
Although in our study Simpson ejection fraction correlated with height, the independent association of preserved cardiac pump function with the risk of aortic aneurysm is supported by the association with other echocardiographic indices, including those reflecting valve function. We should also note the association of aortic aneurysm risk with several laboratory parameters, among which troponin level is the leading one. This index is a known predictor of adverse cardiovascular outcomes [12]. Troponin levels are also associated with abdominal aortic risk [13]. Since troponin, along with D-dimer, is also a marker of acute aortic rupture [14], it can be assumed that in patients with aortic aneurysm the increased troponin level is associated with subclinical damage of the aortic wall, but not of the myocardium.

\section{Conclusion}

We have identified the risk factors for the dynamic development of aortic aneurism. These factors can be obtained from the screening methods that allow early diagnostics of the aneurism development.

\section{Acknowledgements}

This work was financially supported by the Ministry of Science and Higher Education of the Russian Federation (Agreement No.075-15-2020-901).

\section{References}

[1] Neil HAW, Hawkins MM, Durrington PN, Betteridge DJ, Capps NE, Humphries SE. Non-coronary heart disease mortality and risk of fatal cancer in patients with treated heterozygous familial hypercholesterolaemia: a prospective registry study. Atherosclerosis. 2005 Apr;179(2):293-7. doi: 10.1016/j.atherosclerosis.2004.10.011.

[2] Sampson UKA, Norman PE, Fowkes FGR, Aboyans V, Song Y, Harrell FE, et al. Global and regional burden of aortic dissection and aneurysms: mortality trends in 21 world regions, 1990 to 2010. Glob Heart. 2014 Mar;9(1):171-180.e10. doi: 10.1016/j.gheart.2013.12.010.

[3] Devereux RB, De Simone G, Arnett DK, Best LG, Boerwinkle E, et al. Normal limits in relation to age, body size and gender of two-dimensional echocardiographic aortic root dimensions in persons $>15$ years of age. Am J Cardiol. 2012 Oct 15; 110(8): 1189-1194. doi: 10.1016/j.amjcard.2012.05.063

[4] Kälsch H, Lehmann N, Möhlenkamp S, Becker A, Moebus S, et al. Body-surface adjusted aortic reference diameters for improved identification of patients with thoracic aortic aneurysms: Results from the population-based Heinz Nixdorf Recall study. Int J Cardiol. 2013 Feb 10;163(1):72-8. doi: 10.1016/j.ijcard.2011.05.039.

[5] Rogers IS, Massaro JM, Truong QA, Mahabadi AA, Kriegel MF, et al. Distribution, determinants, and normal reference values of thoracic and abdominal aortic diameters by computed tomography (from the framingham heart study). Am J Cardiol. 2013 May 15;111(10):1510-6. doi: 10.1016/j.amjcard.2013.01.306.

[6] Doris MK, Everett RJ, Shun-Shin M, Clavel A-M. Dweck MR. The Role of Imaging in Measuring Disease Progression and Assessing Novel Therapies in Aortic Stenosis. JACC Cardiovasc Imaging. 2019 Jan;12(1):185-197. doi: 10.1016/j.jcmg.2018.10.023.

[7] Evangelista A, Flachskampf FA, Erbel R, Antonini-Canterin F, Vlachopoulos C, et al. Echocardiography in aortic diseases: EAE recommendations for clinical practice. Eur J Echocardiogr. 2010 Sep;11(8):64558. doi: 10.1093/ejechocard/jeq056.

[8] Mądry W, Zacharska-Kokot E, Karolczak MA. Methodology of echocardiographic analysis of morphological variations of the aortic arch and its branches in children - own experience. J Ultrason. 2019;19(76):24-42. doi: 10.15557/JoU.2019.0004. 
[9] Libby P, Buring JE, Badimon L, Hansson GK, Deanfield J, et al. Atherosclerosis, Nature Reviews Disease Primers 2019; 5(56): 1-18. doi:10.1038/s41572-019-0106-Z.

[10] Zafar MA, Li Y, Rizzo JA, Charilaou P, Saeyeldin A, et al. Height alone, rather than body surface area, suffices for risk estimation in ascending aortic aneurysm. J Thorac Cardiovasc Surg. 2018 May;155(5):1938-1950. doi: 10.1016/j.jtcvs.2017.10.140.

[11] Kutyrev K, Yakovlev A, Metsker O. Mortality Prediction Based on Echocardiographic Data and Machine Learning: CHF, CHD, Aneurism, Procedia Computer Science. 2019; 156: 97-104. doi:10.1016/J.PROCS.2019.08.184.

[12] Willeit P, Welch P, Evans JDW, Tschiderer L, Boachie C, et al. High-Sensitivity Cardiac Troponin Concentration and Risk of First-Ever Cardiovascular Outcomes in 154,052 Participants. J Am Coll Cardiol. 2017 Aug 1;70(5):558-568. doi: 10.1016/j.jacc.2017.05.062.

[13] Molacek J, Treska V, Zeithami J, Hollan I, Topolcan O, et al. Blood biomarker panel recommended for personalized prediction, prognosis, and prevention of complications associated with abdominal aortic aneurysm. EPMA J. 2019 Jun 3;10(2):125-135. doi: 10.1007/s13167-019-00173-2.

[14] Li Z-D, Liu Y, Zhu J, Wang J, Lu F-L, Han L, Xu Z-Y. Risk factors of pre-operational aortic rupture in acute and subacute Stanford type A aortic dissection patients. J Thorac Dis. 2017 Dec;9(12):4979-4987. doi: $10.21037 /$ jtd.2017.11.59 